\title{
Urticaria and Angioedema in Pregnancy
}

\author{
Laliv Kadar • Shmuel Kivity
}

Published online: 26 September 2013

(C) Springer Science+Business Media New York 2013

\begin{abstract}
Urticaria and angioedema occur in up to $20 \%$ of the population during their lifetime. Chronic urticaria and some hereditary angioedema states are more frequent in women and fluctuate during the hormonal cycle. This review describes the course of urticarial and angioedema states during pregnancy, as well as a possible role for sex hormones in the pathogenesis of different diseases, both chronic and cyclic urticarial diseases and diseases that occur only in pregnancy.
\end{abstract}

Keywords Urticaria $\cdot$ Angioedema $\cdot$ Pregnancy $\cdot$ Pruritic urticarial papules and plaques of pregnancy $\cdot$ Pemphigoid gestationis $\cdot$ Estrogen $\cdot$ Progesterone $\cdot \mathrm{C} 1$ esterase inhibitor

\section{Introduction}

Urticaria, commonly referred to as hives, appears as raised, well-circumscribed areas of erythema and edema involving the dermis and epidermis and accompanied by pruritus. In classic urticaria, lesions appear on different parts of the body, may confluent, and often resolve without leaving any scars.

Acute urticaria is very common. About $10 \%$ to $20 \%$ of the population experience an urticarial episode at some point during their lives, usually as a result of an allergic reaction, an infection, or direct histamine release by drugs [1]. If the lesions persist for more than 6 weeks, the urticaria is termed chronic, whereas shorter episodes are considered acute. The prevalence of chronic urticaria is $0.5 \%$ to $1 \%$ of the population, and women are affected twice as often as men [2]. The etiology of chronic urticaria may be physical, autoimmune, or idiopathic.

L. Kadar $\cdot$ S. Kivity $(\bowtie)$

Allergy and Immunology Unit, Tel Aviv Sourasky Medical Center, Sackler School of Medicine, Tel Aviv University, Tel Aviv, Israel e-mail: allergy@tlvmc.gov.il
The pathogenesis of urticarial lesions is degranulation of mast cells and the release of vasoactive substances, the most important of which is histamine. This process is mediated by immunoglobulin $\mathrm{E}$ ( $\mathrm{IgE}$ ) binding to the $\mathrm{Fc} \varepsilon$ receptor on mast cells. Other physical, chemical, and hormonal factors may lead to spontaneous degranulation of mast cells, not through an antigen-antibody mechanism [3].

Angioedema is the swelling of deep dermis, subcutaneous, or submucosal tissue due to vascular leakage. It may appear on any part of the body, but if it involves the larynx or oral cavity, it may be life threatening. Although angioedema may accompany urticaria, it occurs alone in $10 \%$ of cases; these cases respond to antihistamine and steroid treatment and therefore are considered histaminergic. In other cases, angioedema may appear as the sole manifestation and last for few days with no response to antihistamines; these cases are termed bradykininergic [4].

There are no published data regarding the incidence of urticaria and angioedema in pregnancy; however, because it occurs in young women, its incidence probably is the same or even greater than in the general population. In this review, we discuss the different etiologies and manifestations of urticaria and angioedema in pregnancy.

\section{Normal Hormonal Changes During Pregnancy}

The human placenta is an endocrine organ, secreting hormones that enable the growth and development of the fetus and the physiologic changes in the maternal body. Among the hormones secreted by the placenta, the steroid hormones (estrogen and progesterone) play a crucial role in maintaining pregnancy. These hormones also may influence the occurrence of urticaria and angioedema during that period.

In the beginning of pregnancy, progesterone is secreted by the corpus luteum, and by the 9th week, the cells of the 
syncytiotrophoblast in the placenta become the main source of this hormone. Progesterone levels gradually peak during the second and third trimesters and reach maximum levels just before delivery. The principle action of this hormone is to support the endometrial tissue during pregnancy and to maintain quiescence of the myometrium, preventing its contractions. It also plays a role in immunomodulatory processes in the fetal-maternal interface.

Estriol, the main estrogen secreted in pregnancy, also is derived from the placenta, and its levels rise gradually to peak toward the end of gestation. Estriol stimulates the growth of the myometrium and antagonizes the myometrial-suppressing activity of progesterone. In many species, the high levels of estrogen in late gestation induce myometrial oxytocin receptors, preparing the uterus for parturition $[5,6]$.

Estrogens and progesterones are considered immunomodulators because of their influence on many cells of the immune system, including CD4+ T cells [7], dendritic cells [8], B cells $[9,10]$, eosinophils [11], and mast cells [12]. Whereas estrogens usually enhance humoral immunity, progesterones and androgens are considered suppressors [13]; both take part in the fluctuation of immune cell function during normal states, such as the menstrual cycle, and in disease states, such as autoimmunity or allergy. For example, some of the classic atopic diseases, such as asthma and atopic dermatitis, are more common in males before puberty and become more common in females after puberty [14]. Both estrogen and progesterone were described as contributors to the development of autoimmune diseases [15], such as rheumatoid arthritis [16] and systemic lupus erythematosus, which primarily occur in females [17].

Pregnancy is a special state in which a suppressive environment is crucial to prevent rejection of the "nonself" tissues of the fetus by the mother. This environment includes high levels of FoxP3+ regulatory T cells and relatively low levels of suppressor cells, natural killer cells, and Th1 lymphocytes in the placenta. Sex hormones play a major role in inducing this environment [18] but also might play a role in diseases during pregnancy.

\section{Urticaria and Hormonal Changes}

Estrogens were found to enhance histamine release from both rat mast cells and human basophils through estrogen receptor- $\alpha$. Progesterone and testosterone inhibit histamine release from mast cells and basophils [19]. Synthetic estrogens, from either unknown origins or environmental pollutants, tend to accumulate in bodily tissues and theoretically can induce rapid histamine release from mast cells. Some studies present this theory as the basis for chronic idiopathic and chronic autoimmune urticaria [20].

There are two specific urticarial dermatoses that occur only in pregnancy: pruritic urticarial plaques of pregnancy (PUPPP) and the first stage of pemphigoid gestationis. Other urticarial rashes that are not pregnancy specific may worsen or flare during pregnancy.

\section{Exacerbations of Existing Urticaria or Angioedema During Pregnancy}

\section{Progesterone Urticaria}

Progesterone urticaria is an autoimmune disease that is part of the spectrum of autoimmune dermatitis, which includes angioedema, erythema multiforme, papulovesicular eruptions, and fixed drug eruptions induced by progesterone. It is characterized by cyclic exacerbations during the luteal phase of the menstrual cycle and is related to fluctuations in serum progesterone levels, which rise a few days after ovulation and fall with the onset of menstruation. Lesions usually occur on the trunk and extremities but rarely may appear on the face or genitalia. Diagnosis often is challenging because it is similar in appearance to other chronic urticarias; however, the cyclic nature and positive skin tests for progesterone distinguish this rash [21].

It is believed that some women treated with progesterone before and during pregnancy for placental insufficiency likely are sensitized to both the synthetic and the biologic compounds. These women might develop progesterone dermatitis or have worsening of their already-existing rash during pregnancy and the peripartum period [22].

Reports on progesterone dermatitis in pregnancy are scarce, most likely because of the remission of disease in this period, which probably is a result of acquired desensitization to the rising levels of progesterone in contrast to the cyclic rise and fall during the menstrual cycle [23]. Nevertheless, a few articles describe the flare of disease during pregnancy. In one case report, progesterone dermatitis was induced by a metabolite of progesterone that was used as supplementation during pregnancy to prevent preterm birth [24]. In another case report, progesterone dermatitis became aggravated during the third trimester of pregnancy, necessitating high doses of steroids [25].

Treatment with antihistamines and steroids for progesterone dermatitis usually is not sufficient. Oral contraceptives that prevent ovulation (and thereby also prevent progesterone increases) are useful, but this treatment is not suitable during pregnancy. Desensitization to synthetic progesterone was reported as successful, especially during in vitro fertilization [26]. In severe cases, oophorectomy is an option [25].

\section{Estrogen-Induced Urticaria}

Estrogen dermatitis is an uncommon disease occurring as cyclic exacerbations of skin eruptions during ovulation and premenstrually. Urticaria is only one of the rashes described in 
this disease [27••]. Patients test positive on intradermal skin testing for estrogen, and they respond to estrogen receptor antagonists such as tamoxifen and progesterone therapy. It is postulated that Langerhans cells play a role in the pathogenesis of disease [28]. There are no reports in the literature about estrogen dermatitis during pregnancy.

\section{Chronic Urticaria During Pregnancy}

Autoimmunity is more frequent in patients with chronic urticaria, which is shown by the increased risk of autoimmune thyroid diseases [29] and the occurrence of IgG antibodies directed to the high-affinity $\mathrm{IgE}$ receptor ( $\mathrm{FC} \varepsilon$ receptor I) on mast cells and basophils or to IgE in $40 \%$ of patients [30]. The high frequency of chronic urticaria in women suggests a possible correlation between sex hormones and the autoimmunity. In one study conducted by Asero [31], about three fourths of female patients with chronic urticaria had positive autologous serum skin tests, which were found to correlate with the autoantibodies mentioned earlier. The frequency in men was only one third. Nevertheless, no direct influence of estrogen or progesterone has ever been demonstrated as a major factor in the pathogenesis of chronic urticaria. A few studies, however, found that patients with chronic urticaria tend to have lower levels of dehydroepiandrosterone sulfate, an androgenic hormone with immunomodulatory functions. It is believed that lower levels of this hormone might contribute to disease flare [32]. Surprisingly, there is no definite pattern for chronic urticaria during pregnancy. Flare-ups may occur in some patients, whereas improvement may occur in others. Albeit the physical changes occurring in the female body during pregnancy, there are no case reports concerning the worsening of physical urticaria during that period.

\section{Hereditary Angioedema in Pregnancy}

\section{Types I and II Hereditary Angioedema}

Hereditary angioedema (HAE) is a rare disease $(1: 10,000$ $50,000)[33]$ in which episodes of edema of the skin and mucous membranes develop. Episodes may last 2 to 5 days and resolve spontaneously, but if they involve the larynx or oral cavity, they may lead to airway obstruction and death. However, the most common site of involvement is the abdomen [34].

The pathogenesis of HAE includes either deficiency (type I) or malfunction (type II) of C1 esterase inhibitor (C1EI), which is a key enzyme in three different pathways:

- the coagulation/fibrinolysis pathway, in which it inhibits factor XII, factor XI, and plasmin
- the classic complement system, in which it inhibits the action of $\mathrm{C} 1 \mathrm{~s}$ and $\mathrm{C} 1 \mathrm{r}$, preventing overactivation of the complement cascade

- the contact system, in which it inhibits the enzyme kallikrein, which forms bradykinin from high molecular weight kininogen

In patients with $\mathrm{HAE}$, the fibrinolysis and complement pathways may compensate for the lack of C1EI, so its deficiency does not lead to clinical manifestations. However, overactivation of kallikrein in the contact system is responsible for bradykinin accumulation in the blood and tissues of patients and to the vasodilatatory effect that leads to edema [35].

Whereas type I HAE occurs in $85 \%$ of cases, type II occurs in only $15 \%$. More than 300 mutations already have been described on the gene for C1EI, on chromosome 11, and the disease is inherited in an autosomal dominant fashion. Twenty-five percent of cases are sporadic, and some carriers never develop the disease [36॰].

The main trigger for an attack is tissue damage, which probably activates the coagulation, complement, and contact systems. For example, dental repair might lead to perioral swelling, whereas an upper respiratory tract infection may result in laryngeal edema [37]. Other triggers to the disease include drugs (e.g., angiotensin-converting enzyme [ACE] inhibitors) and hormonal changes. Although HAE is transferred in an autosomal dominant fashion, penetrance is increased in females [34], and the course of their disease is more severe [38].

Estrogen has been shown to affect the contact and coagulation systems by increasing the levels and function of factor XII, prekallikrein, kallikrein, and high molecular weight kininogen, all of which are involved in the process of bradykinin formation. Estrogens also up-regulate the expression and potentiate the activity of bradykinin receptor $\mathrm{B}_{2}$ [39]. The influence of progesterone on the course of HAE is inconclusive. It is known to raise the levels of kallikrein, thereby increasing bradykinin formation. In one study, it was found that high levels $(>4 \mathrm{nmol} / \mathrm{L})$ of progesterone in nonpregnant women was related to a higher frequency of attacks [40]. In other studies, however, progesterone-containing contraceptives were found to ameliorate the course of disease [41•].

The attack rate may increase, decrease, or remain the same during pregnancy. Bouillet et al. [41•] reported an increase in exacerbations during pregnancy in $38 \%$ of women, a decrease in $30 \%$, and no change in $32 \%$. In the international guidelines concerning gynecologic and obstetric management of female patients with HAE [42••], a higher attack rate was reported in the first trimester of pregnancy [40, 43, 44•]. Some reports also present an increase in attack rates in the second [44•] or third trimester [45 ${ }^{\circ}$. It is believed that the rapid elevation in estrogen levels at the beginning and at the end of pregnancy and the increase in prolactogenic hormones are responsible for 
this rise, whereas the balance between estrogen and progesterone in the second trimester decreases the rate of attacks. The cessation of chronic medications such as danazole during pregnancy is mandatory because of its virilizing effect on the fetus, but it often leads to exacerbations. During pregnancy, attacks occur more frequently in the abdomen, sometimes mimicking uterine contractions and surgical emergencies [45•]. In several case reports, a higher attack rate also was observed during puerperium [46].

Delivery itself, however, did not provoke attacks, except for rare local swelling of the genitalia [47], and there were no more caesarian sections in patients with HAE than in the normal population [48]. Some studies claimed that symptomatic women with HAE experience more spontaneous abortions and premature labor [49], whereas others did not find any evidence for this $[45 \cdot, 48]$.

Factors associated with a more aggressive course in pregnancy include early onset of disease in life, bearing a fetus with C1EI deficiency, and disease exacerbation during menses [39]. According to some studies, the course of disease during a previous pregnancy might predict the clinical picture in subsequent pregnancies $[41 \bullet]$.

\section{Type III HAE}

In 2000, a new type of HAE was described by Bork et al. [50]. This type is a familial angioedema in which more than $90 \%$ of patients are women and both the level and function of C1EI are normal. Typically, the disease does not occur in childhood but occurs after the second decade of life with infrequent attacks, which usually involve the face, tongue, and larynx $[51 \bullet \cdot]$.

So far, two missense mutations (Thr309Arg, Thr309Lys) and one deletion of 72 base pairs have been described in the gene to factor XII and by a yet unknown mechanism that potentiates the contact pathway and probably increases bradykinin production, leading to angioedema. Inheritance is autosomal dominant with incomplete penetrance [52, 53].

As in type I/II HAE, trauma or pressure on the skin might generate an attack. In type III HAE, however, estrogen as a trigger is much more prominent than in type I/II [50, 54], although in some patients, estrogen had a partial or no impact on symptoms [55]. Over $62 \%$ of patients with angioedema and normal C1EI levels reported either worsening or induction of symptoms by estrogens [56]. In another case series, 17 of 27 women reported the occurrence of the first attack shortly after starting oral contraceptives containing estrogens.

Estrogens were proved to reduce plasma $\mathrm{C} 1$-inhibitor levels $[51 \bullet \cdot$ and to increase the transcription of factor XII, plasma prekallikrein, and bradykinin 2 receptors. Estrogens also decrease the levels of ACE inhibitor, a key enzyme in the degradation of bradykinin [39].
Surprisingly, pregnancy has a variable impact on the course of HAE type III. In one study, pregnancy was the trigger for the first attack in 3 of 25 cases, and in 7 women, attacks became more frequent and severe [57•.0. In another case, pregnant women bearing the mutation developed severe complications, including fetal death [58]. In other cases, pregnancy did not lead to exacerbations. Because it is a rare disease, not enough information is known about its course during pregnancy.

\section{Acute Treatment During Pregnancy}

Unlike in chronic urticaria and angioedema, histamine does not play a role in the pathogenesis of the disease. Therefore, urticarial rashes and pruritus are lacking and patients do not respond to antihistamines or steroids $[51 \cdot \bullet]$.

C1EI concentrates, given intravenously, are the drug of choice for acute attacks of type I/II HAE in pregnancy [44•, $45 \cdot 59 \cdot 0]$. They were found to be safe during pregnancy, with no increase in fetal anomalies [44•, 45•], although no controlled studies have been performed in pregnant women. C1EI concentrate also may be given before procedures such as amniocentesis and chorionic villi sampling. There is no indication for its regular use before delivery, but it should be kept in the delivery room and be given in cases of traumatic labor, such as forceps or vacuum use; if intubation is needed; or if the patient has a history of severe attacks during the third trimester [42••]. Treatment with C1EI concentrate in women with type III HAE was found to be effective in some patients [55, 57••] and ineffective in others [54]. There are no data concerning treatment during pregnancy.

Other well-known medications for HAE, such as bradykinin receptor antagonist (icatibant) and kallikrein inhibitor (ecallantide), are not approved for use in pregnancy because there is little information about their teratogenic potential. They should be stopped 1 week before attempting conception $[42 \bullet \bullet]$.

\section{Specific "Urticarial-Like" Dermatoses of Pregnancy}

Although urticarial-like rashes are not pure urticarias, their lesions may have urticarial characteristics during the disease course.

\section{PUPPP}

PUPPP is a benign, self-limited rash that usually occurs in women in the late third trimester of pregnancy; it consists of urticarial papules and plaques, accompanied by intense pruritus [60]. It is the most common dermatosis of pregnancy, occurring in 1 in 160 pregnancies, usually in the first pregnancy, and more often in twin/triplet pregnancies and in obese patients. The rash usually occurs within striae and spreads to 
the extremities, where it may become confluent with urticarial plaques. Involvement of the palms, soles, or face is uncommon [60]. Skin biopsy, although unnecessary for diagnosis, reveals nonspecific changes with perivascular lymphocytic infiltrate, mostly $\mathrm{T}$ helper cells with scarce eosinophils or neutrophils. Dermal immunofluorescence usually is negative [22], although in some patients dermal immunofluorescence is positive for a granular pattern because of deposits of $\operatorname{IgA} / \operatorname{IgM}$ and $\mathrm{C} 3$ in the dermal-epidermal junction [61].

Although the pathogenesis is unknown, it may be related to mechanical factors such as stretching of the abdomen or to maternal exposure to fetal antigens $[62,63]$. A role has been suggested for sex hormones, especially progesterone, which was shown to aggravate inflammation at the tissue level. Increased reactivity of progesterone receptors has been seen in skin lesions of PUPPP [64].

The rash and pruritus gradually resolve after delivery. Treatment is symptomatic and includes low- to mid-potency steroidal creams. The rash does not tend to recur in subsequent pregnancies [60].

\section{Pemphigoid Gestationis}

Pemphigoid gestationis is a rare disease that occurs in 1 in 50,000 to 60,000 pregnancies. It begins in the second or third trimester as pruritus, typically around the umbilicus, followed by a papular urticarial rash with erythematous plaques on the abdomen, trunk, limbs, and back. The mucous membranes and face are spared. After 1 to 2 weeks, large blisters develop [22]. Direct immunofluorescence is positive for $\mathrm{C} 3$ in the basal membrane zone and, in some cases, also positive for IgG1. An immune response to placental matrix antigen, which cross-reacts with skin antigens, is suspected to be the main pathophysiologic feature of disease, and such a protein was identified as bullous pemphigoid antigen 2 [65]. Disease recurrence in women using oral contraceptives in $20 \%$ to $50 \%$ of cases and the potential worsening during the premenstrual period support the theory that hormonal factors also play an essential role in the pathogenesis of the disease [66, 67]. Treatment is with potent corticosteroid ointments, and rarely with oral steroids, combined with antihistamines. The prognosis for the mother is good, although the disease tends to recur in subsequent pregnancies [22].

\section{Conclusions}

Estrogen and progesterone levels, which rise dramatically in pregnancy, probably play a role in at least some urticarial rashes and angioedema states. The influence of estrogens on bradykininergic angioedema of HAE type I/II is the most prominent, and they might aggravate the disease, especially in the first and last trimesters of pregnancy. HAE attacks during pregnancy are more common in the abdomen, probably because of the enormous changes in the uterus and microtrauma during fetal development. Factors associated with a more aggressive course in pregnancy include a disease onset early in life, bearing a fetus with C1EI deficiency, and disease exacerbation during menses. Delivery in patients with HAE type I/II usually is uneventful, and C1EI concentrate is not indicated routinely. Pregnancy has a variable impact on the course of HAE type III. Progesterone and estrogen dermatitis, which tends to fluctuate during the menstrual cycle, usually remits during pregnancy, although exacerbation is described in some case reports. Chronic urticaria is unlikely to flare in pregnancy. Special dermatoses of pregnancy include PUPPP and pemphigoid gestationis, with urticarial-like lesions that occur during the second or third trimester of pregnancy and resolve after delivery. Sex hormones might play some role in the pathogenesis of disease.

\section{Compliance with Ethics Guidelines}

Conflict of Interest Ialiv Kadar declares no conflicts of interest. Shmuel Kivity declares no conflicts of interest.

Human and Animal Rights and Informed Consent This article does not contain any studies with human or animal subjects performed by any of the authors.

\section{References}

Papers of particular interest, published recently, have been highlighted as:

- Of importance

-. Of major importance

1. Kaplan AP. In: Adkinson Jr NF, Busse WW, Bochner BS, et al., editors. Middleton's allergy: principles and practice. 7 th ed. Philadelphia: Elsevier; 2009. p. 1064-7.

2. Greaves M. Chronic urticaria. J Allergy Clin Immunol. 2000;105(4):664.

3. Carr TF, Saltoun CA. Chapter 21: urticaria and angioedema. Allergy Asthma Proc. 2012;33 Suppl 1:S70-2.

4. Saltoun CA. Urticaria, angioedema and hereditary angioedema. In: Grammer LC, Greenberger PA, editors. Patterson's allergic diseases. 7th ed. Philadelphia: Lippincott Williams and Wilkins; 2009. p. 539-53.

5. Burton GJ, Sibly CP, Jauniaux E. Placental anatomy and physiology. In: Gabbe SG, Niebyl JR, Galan HL, et al., editors. Obstetrics: normal and problem pregnancies. 6th ed. Philadelphia: Elsevier; 2012. p. 17-9.

6. Cunningham FG, Leveno KL, Bloom SL, et al. Implantation, embryogenesis, and placental development. In: Williams obstetrics, 23rd edn. New York: McGraw-Hill; 2010.

7. Matsuzaki J, Tsuji T, Imazeki I, et al. Immunosteroid as a regulator for Th1/Th2 balance: its possible role in autoimmune diseases. Autoimmunity. 2005;38(5):369-75.

8. Hughes GC, Clark EA. Regulation of dendritic cells by female sex steroids: relevance to immunity and autoimmunity. Autoimmunity. 2007;40:470-81.

9. Sakiani S, Olsen NJ, Kovacs WJ. Gonadal steroids and humoral immunity. Nat Rev Endocrinol. 2013;9(1):56-62. 
10. Incorvaia E, Sicouri L, Petersen-Mahrt SK, Schmitz KM. Hormones and AID: balancing immunity and autoimmunity. Autoimmunity. 2013;46(2):128-37.

11. Hamano N, Terada N, Maesako K, Numata T, Konno A. Effect of sex hormones on eosinophilic inflammation in nasal mucosa. Allergy Asthma Proc. 1998;19:263-9.

12. Zierau O, Zenclussen AC, Jensen F. Role of female sex hormones, estradiol and progesterone, in mast cell behavior. Front Immunol. 2012;3:169.

13. Tanriverdi F, Silveira LF, MacColl GS, Bouloux PM. The hypothalamic-pituitary gonadal axis: immune function and autoimmunity. J Endocrinol. 2003;176(3):293-304.

14. Chen W, Mempel M, Schober W, et al. Gender difference, sex hormones, and immediate type hypersensitivity reactions. Allergy. 2008;63(11):1418-27.

15. Robinson DP, Klein SL. Pregnancy and pregnancy-associated hormones alter immune responses and disease pathogenesis. Horm Behav. 2012;62(3):263-71.

16. Tedeschi SK, Bermas B, Costenbader KH. Sexual disparities in the incidence and course of SLE and RA. Autoimmunity. 2012;45(5): 364-76.

17. Cutolo M, Sulli A, Capellino S, et al. Sex hormones influence on the immune system: basic and clinical aspects in autoimmunity. Lupus. 2004;13(9):635-8.

18. Xiong YH, Yuan Z, He L. Effects of estrogen on CD4(+) CD25(+) regulatory $\mathrm{T}$ cell in peripheral blood during pregnancy. Asian Pac J Trop Med. 2013;6(9):748-52.

19. Zaitsu M, Narita S, Lambert KC, et al. Estradiol activates mast cells via an ongenomic estrogen receptor-alpha and calcium influx. Mol Immunol. 2007;44:1977-85.

20. Narita S, Goldblum RM, Watson CS, et al. Environmental estrogens induce mast cell degranulation and enhance IgE-mediated release of allergic mediators. Environ Health Perspect. 2007;115: $48-52$.

21. Vasconcelos C, Xavier P, Vieira AP, et al. Autoimmune progesterone urticaria. Gynecol Endocrinol. 2000;14(4):245-7.

22. Roth MM. Pregnancy dermatoses: diagnosis, management, and controversies. Am J Clin Dermatol. 2011;12(1):25-41.

23. Toms-Whittle LM, John LH, Griffiths DJ, Buckley DA. Autoimmune progesterone dermatitis: a diagnosis easily missed. Clin Exp Dermatol. 2011;36(4):378-80.

24. Bandino JP, Thoppil J, Kennedy JS, Hivnor CM. Iatrogenic autoimmune progesterone dermatitis caused by 17 alpha-hydro xyprogesterone caproate for preterm labor prevention. Cutis. 2011;88(5):241-3.

25. O'Rourke J, Khawaja N, Loughrey J, McKenna P. Autoimmune progesterone dermatitis in a parturient for emergency caesarean section. Int J Obstet Anesth. 2004;13(4):275-8.

26. Prieto-Garcia A, Sloane DE, Gargiulo AR, et al. Autoimmune progesterone dermatitis: clinical presentation and management with progesterone desensitization for successful in vitro fertilization. Fertil Steril. 2011;95(3):1121.

27. •- Kasperska-Zajac A, Brzoza Z, Rogala B. Sex hormones and urticaria. J Dermatol Sci. 2008;52(2):79-86. An excellent article that reviews the current interaction of estrogen and progesterone with urticaria in women.

28. Yotsumoto S, Shimomai K, Hashiguchi T, et al. Estrogen dermatitis: a dendritic-cell-mediated allergic condition. Dermatology. 2003;207(3): 265-8.

29. Zauli D, Deleonardi G, Foderaro S, et al. Thyroid autoimmunity in chronic urticaria. Allergy Asthma Proc. 2001;22(2):93-5.

30. Hide M, Francis DM, Grattan CEH, et al. Autoantibodies against the high affinity $\operatorname{IgE}$ receptor as a cause of histamine release in chronic urticaria. N Engl J Med. 1993;328:1599-604.

31. Asero R. Sex differences in the pathogenesis of chronic urticaria. J Allergy Clin Immunol. 2003;111(2):425-6.
32. Kasperska-Zajac A, Brzoza Z, Rogala B. Lower serum concentration of dehydroepiandrosterone sulphate in patients suffering from chronic idiopathic urticaria. Allergy. 2006;61(12):1489-90.

33. Cicardi M, Agostoni A. Hereditary angioedema. N Engl J Med. 1996;334:1666.

34. Agostoni A, Cicardi M. Hereditary and acquired C1-inhibitor deficiency: biological and clinical characteristics in 235 patients. Medicine (Baltimore). 1992;71:206-15.

35. Georgy MS, Pongracic JA. Chapter 22: hereditary and acquired angioedema. Allergy Asthma Proc. 2012;33 Suppl 1:S73-6.

36. - Agostoni A, Aygören-Pürsün E, Binkley KE, et al. Hereditary and acquired angioedema: problems and progress: proceedings of the third $\mathrm{C} 1$ esterase inhibitor deficiency workshop and beyond. J Allergy Clin Immunol. 2004;114(3 Suppl):S51-S131. General review on HAE: diagnosis, pathogenesis and treatment in different populations including pregnant women.

37. Agostoni A, Aygoren-Pursun E, Binkley KE, et al. Clinical studies of sudden upper airway obstruction in patients with hereditary angioedema due to $\mathrm{C} 1$ esterase inhibitor deficiency. Arch Intern Med. 2003;163:1229-35.

38. Zilberberg MD, Nathanson BH, Jacobsen T, Tillotson G. Descriptive epidemiology of hereditary angioedema hospitalizations in the United States, 2004-2007. Allergy Asthma Proc. 2011;32(3): 248-54.

39. Geng B, Riedl MA. HAE update: special considerations in the female patient with hereditary angioedema. Allergy Asthma Proc. 2013;34(1):13-8.

40. Visy B, Füst G, Varga L, et al. Sex hormones in hereditary angioneurotic oedema. Clin Endocrinol (Oxf). 2004;60(4):508-15.

41. • Bouillet L, Longhurst H, Boccon-Gibod I, et al. Disease expression in women with hereditary angioedema. Am J Obstet Gynecol. 2008;199(5):484. Although a retrospective study, it is a part of the PREHAEAT Project on 150 type I HAE women that speculates different phenotypes of hormonal sensitivity in women patients.

42. • Caballero T, Farkas H, Bouillet L, C-1-INH Deficiency Working Group, et al. International consensus and practical guidelines on the gynecologic and obstetric management of female patients with hereditary angioedema caused by $\mathrm{C} 1$ inhibitor deficiency. J Allergy Clin Immunol. 2012;129(2):308-20. Evidence based guidelines for the management of HAE in pregnancy and the peripartum period.

43. Chinniah N, Katelaris $\mathrm{CH}$. Hereditary angioedema and pregnancy. Aust N Z J Obstet Gynaecol. 2009;49(1):2-5.

44. • Martinez-Saguer I, Rusicke E, Aygören-Pürsün E, et al. Characterization of acute hereditary angioedema attacks during pregnancy and breast-feeding and their treatment with $\mathrm{C} 1$ inhibitor concentrate. Am J Obstet Gynecol. 2010;203(2):131e1-7. A prospective study important for describing risk factors for increased attack rate in $H A E$ patients during pregnancy.

45. - Czaller I, Visy B, Csuka D, et al. The natural history of hereditary angioedema and the impact of treatment with human $\mathrm{C} 1$-inhibitor concentrate during pregnancy: a long-term survey. Eur J Obstet Gynecol Reprod Biol. 2010;152(1):44-9. A prospective study that emphasizes the safety of $C 1$ esterase inhibitor treatment in 91 cases during pregnancy.

46. Cunningham DS, Jensen JT. Hereditary angioneurotic edema in the puerperium. A case report. J Reprod Med. 1991;36(4):312-3.

47. Cox M, Holdcroft A. Hereditary angioneurotic oedema: current management in pregnancy. Anaesthesia. 1995;50:534-49.

48. Bouillet L, Longhurst H, Boccon-Gibod I, et al. Disease expression in women with hereditary angioedema. Am J Obstet Gynecol. 2008;199(5):484.e1-4.

49. Nielsen EW, Gran JT, Straume B, et al. Hereditary angio-oedema: new clinical observations and autoimmune screening, complement and kallikrein-kinin analyses. J Intern Med. 1996;239(2):119-30.

50. Bork K, Barnstedt SE, Koch P, Traupe H. Hereditary angioedema with normal C1-inhibitor activity in women. Lancet. 2000;356(9225):213-7. 
51. • Zuraw BL, Bork K, Binkley KE, et al. Hereditary angioedema with normal $\mathrm{C} 1$ inhibitor function: consensus of an international expert panel. Allergy Asthma Proc. 2012;33 Suppl 1:S145-56. A recently published internal panel consensus defining the diagnostic criteria, clinical characteristics, diagnosis and possible treatment for type III HAE.

52. Bork K, Gül D, Hardt J, Dewald G. Hereditary angioedema with normal C1 inhibitor: clinical symptoms and course. Am J Med. 2007;120(11):987-92.

53. Bork K, Wulff K, Meinke P, et al. A novel mutation in the coagulation factor 12 gene in subjects with hereditary angioedema and normal C1-inhibitor. Clin Immunol. 2011;141(1):31-5.

54. Binkley KE, Davis 3rd A. Clinical, biochemical, and genetic characterization of a novel estrogen-dependent inherited form of angioedema. J Allergy Clin Immunol. 2000;106(3):546-50.

55. Vitrat-Hincky V, Gompel A, Dumestre-Perard C, et al. Type III hereditary angio-oedema: clinical and biological features in a French cohort. Allergy. 2010;65(10):1331-6.

56. Bork K, Fischer B, Dewald G. Recurrent episodes of skin angioedema and severe attacks of abdominal pain induced by oral contraceptives or hormone replacement therapy. Am J Med. 2003;114(4):294-8.

57. • Bork K, Wulff K, Hardt J, et al. Hereditary angioedema caused by missense mutations in the factor XII gene: clinical features, trigger factors, and therapy. J Allergy Clin Immunol. 2009;124(1):129-34. An excellent review of the leading group that described first and investigates patients with HAE type III.

58. Picone O, Donnadieu AC, Brivet FG, et al. Obstetrical complications and outcome in two families with hereditary angioedema due to mutation in the F12 gene. Obstet Gynecol Int. 2010;2010:957507.
59. •• Bowen T, Cicardi M, Farkas H, et al. 2010 international consensus algorithm for the diagnosis, therapy and management of hereditary angioedema. Allergy Asthma Clin Immunol. 2010;6(1):24. Canadian consensus algorithm that presents flowcharts for the diagnosis and treatment of acute attacks and preventive treatment of all 3 types of $H A E$.

60. Matz H, Orion E, Wolf R. Pruritic urticarial papules and plaques of pregnancy: polymorphic eruption of pregnancy (PUPPP). Clin Dermatol. 2006;24(2):105-8.

61. Trattner A, Ingber A, Sandbank M. Antiepidermal cell surface antibodies in a patient with pruritic urticarial papules and plaques of pregnancy. J Am Acad Dermatol. 1991;24(2 Pt 1):306-8.

62. Yancey KP, Hall RP, Lawley TJ. Pruritic urticarial papules and plaques of pregnancy. Clinical experience in twenty-five patients. $J$ Am Acad Dermatol. 1984;10:473-80.

63. Cohen LM, Capeless EL, Krusinski PA, et al. Pruritic urticarial papules and plaques of pregnancy and its relationship to maternalfetal weight gain and twin pregnancy. Arch Dermatol. 1989;125: 1534-6.

64. Im S, Lee E-S, Kim W, et al. Expression of progesterone receptor in human keratinocytes. J Korean Med Sci. 2000;15:647-54.

65. Zillikens D. Pemphigoid gestationis: immunological aspects. J Eur Acad Dermatol Venereol. 2004;18 Suppl 2:6-42.

66. Schirren H, Messer G, Schirren CG, Meurer M. Immunogenetic findings in herpes gestationis. Hautarzt. 1993;44(12):767-71.

67. Jenkins RE, Shornick J. Pemphigoid gestationis. In: Black MM, Ambros-Rudolph C, Edwards L, et al., editors. Obstetric and gynecologic dermatology. 3rd ed. London: Mosby; 2008. p. 37-47. 